\title{
Study on Influence of Diabetes Mellitus for the Charged Cost and Length of Stay among the Angina Pectoris Patient in Japan
}

\author{
Shinya Matsuda'), Tatsuhiko Kubo'), Yoshihisa Fujino' ${ }^{1)}$, Atsuhiko Murata ${ }^{1)}$,

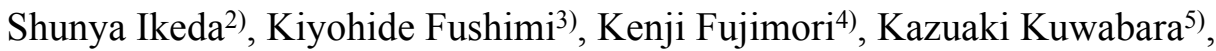 \\ Hideo Yasunaga ${ }^{6}$, Hiromasa Horiguchi'), Koichi Benjamin Ishikawa ${ }^{7)}$ \\ 1) Department of Preventive Medicine and Community Health, University of Occupational and Environmental \\ Health \\ 2) Department of Pharmaceutical Sciences, International University of Health and Welfare \\ 3) Department of Health Policy and Informatics, Tokyo Medical and Dental University Graduate School of Medicine \\ 4) Division of Medical Management, Hokkaido University Hospital \\ ${ }^{5)}$ Graduate School of Medical Sciences, Department of Health Care Administration and Management, Kyushu \\ University \\ 6) Health Management and Policy, Graduate School of Medicine, Tokyo University \\ 7) Statistics and Cancer Control Division, National Cancer Center
}

\begin{abstract}
In order to clarify the importance of diabetes control, the authors examine the impact of diabetes mellitus on resource consumption among the angina pectoris patient institutionalized in the Japanese acute hospitals. We used the 2009 DPC database. The DPC database is a discharge summary and administrative claims database that represents approximately 50\% inpatient admissions to acute care hospitals in Japan. Among 2,553,283 acute in-patient cases of 855 hospitals, we extracted 115,868 patients with the diagnosis of angina pectoris (DPC code with six digits $=050050$ ). Based on this dataset, we compared the resource consumption measured by length of stay and charged cost between the angina pectoris patients with diabetes and those without diabetes. The diabetic patients showed lower mean ages (male: 68.1 vs. 67.2 , female: 73.0 vs. 71.4), longer length of stay (male: 6.4 vs. 6.9 , female: 7.6 vs. 8.3 ), higher charged cost (male: $140,314.0$ vs. $145,206.9$, female: $143,305.1$ vs. 149,252.1; 1 point $=10 \mathrm{JPY}$ ) and higher CCI (male: 1.3 vs. 1.6, female: 1.3 vs.1.5) with statistical significances. Furthermore, there was a wide regional variation among the diabetes prevalence among the observed population. The present analysis has clarified that diabetes increase the resource consumption among the angina pectoris patients. In order to control the health expenditures and ameliorate the population's health status, the preventive activity of diabetes must be put more emphasis.
\end{abstract}

Key words: diabetes mellitus, angina pectoris, DPC, resource consumption

Received: June 2, 2010

Accepted: September 29, 2010

Correspondence: S. Matsuda, Department of Preventive Medicine and Community Health, University of Occupational and Environmental Health, 1-1 Iseigaoka, Yahatanishiku, Kitakyushu, Fukuoka 807-8555, Japan e-mail: smatsuda@med.uoeh-u.ac.jp

\section{* Introduction}

Along with the socio-economic development, the Japanese disease structure has changed from the acute diseases dominant to the life-style related chronic diseases dominant pattern. The life style related diseases account for two-thirds of total deaths, and one third of 
health expenditures in Japan ${ }^{1,2)}$. As other developed countries, the incidence of ischemic heart diseases (IHD) has been increasing. According to the Patient survey in 2008, there were 406 thousands IHD patients who received medical treatments during the survey periods ( 3 days at the end of October) in $\mathrm{Japan}^{3}$. Because of the advanvce in medical technology, most of the ischemic heart diseases patients can survive after the acute event that was formaerly fatal. However, the advance in survival rate causes the increase of chronic heart failure patients. Thus it becomes very impoprtant to prevent the ischemic heart diseases patients in order to ameliorate the population's health and at the same to control the health expenditures.

Behind the rapid increase of IHD, there is a problem of the increase of diabetes patients. According to the result of 2008 National Diebetes Suvey, it is estimated that 18.7 million persons had diabetes mellitus or its possibility). Today diabetes becomes one of the biggest threats for public health in Japan.

In order to clarify the importance of diabetes control, the authors have examined the impact of diabetes mellitus on resource consumption among the angina pectoris patients who were institutionalized in the Japanese acute hospitals based on the Diagnosis Procedure Combinations (DPC) data of 2008.

\section{Methods}

\section{The DPC database}

The DPC database is a discharge summary and administrative claims database that represents approximately $50 \%$ inpatient admissions to acute care hospitals in Japan ${ }^{5)}$. Data are compiled between 1 July and 31 December 2008 by the DPC Research Group funded by the Ministry of Health, Labour and Welfare, Japan. The total number of cases was 2,553,283 from 855 hospitals. For this study, we extracted 115,868 patients with the diagnosis of angina pectoris (DPC code with six digits $=050050$ ).

The database includes the following data: location of hospitals; date of admission and discharge; patients' age and sex; diagnoses, comorbidities and complications; drugs and devices used; procedures; lengths of stay and charged base cost (Fee for Service equivalent). In the DPC database, the diagnoses were recorded with the International Classification of Diseases, Tenth Revision (ICD-10) codes and text data in
Japanese language.

Under the DPC scheme, the payment for hospitals composes of two components; DPC component and Fee-For-Service component. The DPC component corresponds to the "so called" hospital fee, which contains hotel fee, pharmaceuticals and supplies used in wards, lab-test, radiological examination, and procedures cheaper than $¥ 10,000$ (US\$ $100 ; 1 \mathrm{USD}=100$ JPY). The FFS component corresponds to tariffs for surgical procedures and anesthesia, pharmaceuticals and expensive devices used in operation rooms, and procedures more than $¥ 10,000$ (US\$100). For the DPC component, per diem payment schedule is set for each DPC group. In this analysis, we did not use the bundled prices for the calculation of cost. We estimated a fee-for-service charged cost based on the detailed process data that was collected in the DPC research program.

\section{Analyses}

At first, we performed the descriptive analyses of studied population about sex, age, DPC coding, prevalence of diabetes mellitus, regional distribution. Then we compared the resource consumption measured by length of stay and charged cost between the angina pectoris patients with diabetes and those without diabetes. In order to make the comparison homogenous, the patients with DPC "050050xx03x0xx (Angina pectoris, percutaneous coronary intervention, no additional intervention)" were used for the comparison because the number of patient with this classification was large enough for the further analyses. The threshold for significance was a $p$ value of less than 0.05 . All the statistical analyses were conducted with the use of the software Statistical Package for Social Sciences (SPSS) version 15.0J (SPSS Inc., Tokyo, Japan).

Study approval was obtained from our institutional review board (University of Occupational and Environmental Health).

\section{Results}

Table 1 shows basic statistics of studied population. There were 115,868 angina pectoris cases during 1 July to 31 December 2008. Seventy one percent $(82,624)$ were male patients and $24.4 \%(28,262)$ had diabetes as co-morbidity. So far as DPC classifications, "050050xx9910xx (Angina pectoris, no surgi- 
Table 1 Basic statistics of studied population

\begin{tabular}{|c|c|c|}
\hline Sex & $\mathrm{N}$ & $\%$ \\
\hline Male & 82,624 & 71.3 \\
\hline Female & 33,244 & 28.7 \\
\hline $\mathrm{DPC}$ & $\mathrm{N}$ & $\%$ \\
\hline $050050 \times x 01 \times 0 \times x$ & 23 & 0.0 \\
\hline $050050 \times x 01 \times 2 \times x$ & 6 & 0.0 \\
\hline $050050 \times x 01 \times 4 x x$ & 7 & 0.0 \\
\hline $050050 \times x 02 \times 0 \times x$ & 2,908 & 2.5 \\
\hline $050050 \times x 02 \times 1 \times x$ & 255 & 0.2 \\
\hline $050050 \times x 02 \times 2 \times x$ & 586 & 0.5 \\
\hline $050050 \times x 02 \times 3 \times x$ & 1 & 0.0 \\
\hline $050050 \times x 02 \times 4 \times x$ & 687 & 0.6 \\
\hline $050050 \times x 03 \times 0 \times x$ & 31,955 & 27.6 \\
\hline $050050 \times x 03 \times 10 x$ & 1,363 & 1.2 \\
\hline $050050 \times x 03 \times 11 x$ & 646 & 0.6 \\
\hline $050050 \times x 03 \times 2 \times x$ & 2,688 & 2.3 \\
\hline $050050 \times x 03 \times 3 \times x$ & 14 & 0.0 \\
\hline $050050 \times x 03 \times 4 \times x$ & 652 & 0.6 \\
\hline $050050 \times x 97 \times 0 \times x$ & 534 & 0.5 \\
\hline 050050xx97x1xx & 41 & 0.0 \\
\hline $050050 \times x 97 \times 2 \times x$ & 203 & 0.2 \\
\hline $050050 \times x 97 \times 3 \times x$ & 2 & 0.0 \\
\hline 050050xx97x4xx & 363 & 0.3 \\
\hline 050050xx99000x & 3,147 & 2.7 \\
\hline 050050xx99001x & 1,120 & 1.0 \\
\hline 050050xx9901xx & 122 & 0.1 \\
\hline 050050xx9902xx & 1,392 & 1.2 \\
\hline 050050xx9903xx & 1 & 0.0 \\
\hline 050050xx9910xx & 59,516 & 51.4 \\
\hline 050050xx9911xx & 2,247 & 1.9 \\
\hline 050050xx9912xx & 5,383 & 4.6 \\
\hline 050050xx9913xx & 6 & 0.0 \\
\hline Diabetes & $\mathrm{N}$ & $\%$ \\
\hline No & 87,606 & 75.6 \\
\hline Yes & 28,262 & 24.4 \\
\hline Total & 115,868 & 100.0 \\
\hline
\end{tabular}

9th and 10th digits for surgery

01: Disection of cardiac aneurysm, 02: CABG, 03: PCI, 97: Other surgical procedures, 99: No surgery.

12th digit for additional procedure

0 : No additional prpcedure, 1: Haemodyalysis, SPECT, t-PA, Pacemaking.

13th digit for comorbidities and complications (CC) 0: No CC, 1: Existed CC. cal intervention, with cathetel examination)" was the most frequent (59,516 cases; $51.4 \%)$, followed by "050050xx03x0xx (Angina pectoris, percutaneous coronary intervention, no additional intervention)"; 31,955 cases $(27.6 \%)$. Average of age, length of stay and total charged cost were $68.5 \mathrm{yr}$ old (SD: 10.4), 6.7 days (SD: 9.4) and 78607.9 points (SD: 100364.5, 1 point $=10$ Japanese yen), respectively.

Table 2 shows the prevalence rate of diabetes stratified by sex and prefecture. There were wide regional variations in prevalence. The highest prevalence was observed in Tokushima prefecture for male (43.2\%) and in Mie prefecture for female (38.8\%). The lowest prevalence was observed in Okayama $(11.4 \%)$ for male and in Ehime prefecture for female (8.7\%).

Table 3 shows the outcome at discharge stratified by the existence of diabetes. There was no difference in outcome; $97 \%$ of angina pectoris cases of DPC "050050xx03x0xx" recovered.

Table 4 shows the results of comparison of resource consumption between diabetic cases and non-diabetic ones (DPC "050050xx03x0xx"). The diabetic patients showed lower mean ages (male: 68.1 vs. 67.2 , female: 73.0 vs. 71.4 ), longer length of stay (male: 6.4 vs. 6.9 , female: 7.6 vs. 8.3 ), higher charged cost (male: 140,314.0 vs.145,206.9, female: $143,305.1$ vs. $149,252.1 ; 1$ point $=10 \mathrm{JPY})$ and higher Charlson's Co-morbidity Index (male: 1.3 vs. 1.6, female: 1.3 vs.1.5) with statistical significances. For charged cost, consultation (561.8 vs. 675.6), prescription (1,348.2 vs. $1,588.2)$, injection (759.9 vs. 855.7 ) and surgical intervention $(118,770.3$ vs. $121,949.9)$ were higher for male with statistical significance and consultation (627.1 vs. 789.4), prescription (1,463.6 vs. 1,691.1), and surgical intervention (117,776.7 vs. 121,965.9) were higher for female with statistical significance.

\section{- Discussion}

It is well known that diabetes is one of the most important risk factors for ischemic heart diseases. For example, Almdal et al. reported that the diabetic patients showed 1.5- to 4.5-fold (women) and 1.5- to 2 -fold (men) more possibility to experience the ischemic heart diseases (IHDs) and 1.5 to 2 times more possibility of death ${ }^{6}$. According to the results of present study, the diabetic patients would have IHDs at 
Table 2 Prevalence of diabetes stratified by sex and prefecture

$(\mathrm{DPC}$ code with six digits $=050050)$

\begin{tabular}{|c|c|c|c|c|c|c|c|}
\hline & & & & & & Bo & $\operatorname{sex}$ \\
\hline & & Diabetes & Sub-Total & Diabetes & Sub-Total & Diabetes & Sub-Total \\
\hline 01 & $\mathrm{~N}$ & 1,154 & 4,799 & 492 & 2,098 & 1,646 & 6,897 \\
\hline Hokkaido & $\%$ & 24.0 & 100.0 & 23.5 & 100.0 & 23.9 & 100.0 \\
\hline 02 & $\mathrm{~N}$ & 55 & 431 & 31 & 178 & 86 & 609 \\
\hline Aomori & $\%$ & 12.8 & 100.0 & 17.4 & 100.0 & 14.1 & 100.0 \\
\hline 03 & $\mathrm{~N}$ & 312 & 1,027 & 106 & 365 & 418 & 1,392 \\
\hline Iwate & $\%$ & 30.4 & 100.0 & 29.0 & 100.0 & 30.0 & 100.0 \\
\hline 04 & $\mathrm{~N}$ & 387 & 1,564 & 155 & 697 & 542 & 2,261 \\
\hline Miyagi & $\%$ & 24.7 & 100.0 & 22.2 & 100.0 & 24.0 & 100.0 \\
\hline 05 & $\mathrm{~N}$ & 120 & 560 & 40 & 208 & 160 & 768 \\
\hline Akita & $\%$ & 21.4 & 100.0 & 19.2 & 100.0 & 20.8 & 100.0 \\
\hline 06 & $\mathrm{~N}$ & 108 & 821 & 44 & 303 & 152 & 1,124 \\
\hline Yamagata & $\%$ & 13.2 & 100.0 & 14.5 & 100.0 & 13.5 & 100.0 \\
\hline 07 & $\mathrm{~N}$ & 215 & 759 & 85 & 342 & 300 & 1,101 \\
\hline Fukushima & $\%$ & 28.3 & 100.0 & 24.9 & 100.0 & 27.2 & 100.0 \\
\hline 08 & $\mathrm{~N}$ & 452 & 2,064 & 118 & 633 & 570 & 2,697 \\
\hline Ibaraki & $\%$ & 21.9 & 100.0 & 18.6 & 100.0 & 21.1 & 100.0 \\
\hline 09 & $\mathrm{~N}$ & 291 & 910 & 78 & 280 & 369 & 1,190 \\
\hline Tochigi & $\%$ & 32.0 & 100.0 & 27.9 & 100.0 & 31.0 & 100.0 \\
\hline 10 & $\mathrm{~N}$ & 371 & 1,445 & 142 & 627 & 513 & 2,072 \\
\hline Gunma & $\%$ & 25.7 & 100.0 & 22.6 & 100.0 & 24.8 & 100.0 \\
\hline 11 & $\mathrm{~N}$ & 811 & 2,289 & 289 & 825 & 1,100 & 3,114 \\
\hline Saitama & $\%$ & 35.4 & 100.0 & 35.0 & 100.0 & 35.3 & 100.0 \\
\hline 12 & $\mathrm{~N}$ & 849 & 3,196 & 346 & 1,227 & 1,195 & 4,423 \\
\hline Chiba & $\%$ & 26.6 & 100.0 & 28.2 & 100.0 & 27.0 & 100.0 \\
\hline 13 & $\mathrm{~N}$ & 1,722 & 6,815 & 525 & 2,218 & 2,247 & 9,033 \\
\hline Tokyo & $\%$ & 25.3 & 100.0 & 23.7 & 100.0 & 24.9 & 100.0 \\
\hline 14 & $\mathrm{~N}$ & 1,652 & 6,801 & 605 & 2,788 & 2,257 & 9,589 \\
\hline Kanagawa & $\%$ & 24.3 & 100.0 & 21.7 & 100.0 & 23.5 & 100.0 \\
\hline 15 & $\mathrm{~N}$ & 135 & 504 & 53 & 201 & 188 & 705 \\
\hline Niigata & $\%$ & 26.8 & 100.0 & 26.4 & 100.0 & 26.7 & 100.0 \\
\hline 16 & $\mathrm{~N}$ & 153 & 673 & 69 & 312 & 222 & 985 \\
\hline Toyama & $\%$ & 22.7 & 100.0 & 22.1 & 100.0 & 22.5 & 100.0 \\
\hline 17 & $\mathrm{~N}$ & 341 & 1,393 & 120 & 642 & 461 & 2,035 \\
\hline Ishikawa & $\%$ & 24.5 & 100.0 & 18.7 & 100.0 & 22.7 & 100.0 \\
\hline 18 & $\mathrm{~N}$ & 51 & 184 & 35 & 106 & 86 & 290 \\
\hline Fukui & $\%$ & 27.7 & 100.0 & 33.0 & 100.0 & 29.7 & 100.0 \\
\hline 19 & $\mathrm{~N}$ & 10 & 38 & 4 & 24 & 14 & 62 \\
\hline Yamanashi & $\%$ & 26.3 & 100.0 & 16.7 & 100.0 & 22.6 & 100.0 \\
\hline 20 & $\mathrm{~N}$ & 250 & 964 & 95 & 418 & 345 & 1,382 \\
\hline Nagano & $\%$ & 25.9 & 100.0 & 22.7 & 100.0 & 25.0 & 100.0 \\
\hline 21 & $\mathrm{~N}$ & 441 & 2,633 & 163 & 1,083 & 604 & 3,716 \\
\hline Gifu & $\%$ & 16.7 & 100.0 & 15.1 & 100.0 & 16.3 & 100.0 \\
\hline 22 & $\mathrm{~N}$ & 306 & 1,990 & 116 & 751 & 422 & 2,741 \\
\hline Shizuoka & $\%$ & 15.4 & 100.0 & 15.4 & 100.0 & 15.4 & 100.0 \\
\hline 23 & $\mathrm{~N}$ & 676 & 3,748 & 259 & 1,370 & 935 & 5,118 \\
\hline Aichi & $\%$ & 18.0 & 100.0 & 18.9 & 100.0 & 18.3 & 100.0 \\
\hline 24 & $\mathrm{~N}$ & 388 & 1,104 & 153 & 394 & 541 & 1,498 \\
\hline Mie & $\%$ & 35.1 & 100.0 & 38.8 & 100.0 & 36.1 & 100.0 \\
\hline
\end{tabular}


Table 2 Prevalence of diabetes stratified by sex and prefecture

$(\mathrm{DPC}$ code with six digits $=050050)$

\begin{tabular}{|c|c|c|c|c|c|c|c|}
\hline & & & & & & Bo & sex \\
\hline & & Diabetes & Sub-Total & Diabetes & Sub-Total & Diabetes & Sub-Total \\
\hline 25 & $\mathrm{~N}$ & 281 & 1,309 & 121 & 575 & 402 & 1,884 \\
\hline Shiga & $\%$ & 21.5 & 100.0 & 21.0 & 100.0 & 21.3 & 100.0 \\
\hline 26 & $\mathrm{~N}$ & 263 & 1,534 & 109 & 613 & 372 & 2,147 \\
\hline Kyoto & $\%$ & 17.1 & 100.0 & 17.8 & 100.0 & 17.3 & 100.0 \\
\hline 27 & $\mathrm{~N}$ & 2,262 & 7,915 & 803 & 2,931 & 3,065 & 10,846 \\
\hline Osaka & $\%$ & 28.6 & 100.0 & 27.4 & 100.0 & 28.3 & 100.0 \\
\hline 28 & $\mathrm{~N}$ & 719 & 3,477 & 274 & 1,229 & 993 & 4,706 \\
\hline Hyogo & $\%$ & 20.7 & 100.0 & 22.3 & 100.0 & 21.1 & 100.0 \\
\hline 29 & $\mathrm{~N}$ & 138 & 762 & 58 & 319 & 196 & 1,081 \\
\hline Nara & $\%$ & 18.1 & 100.0 & 18.2 & 100.0 & 18.1 & 100.0 \\
\hline 30 & $\mathrm{~N}$ & 141 & 457 & 63 & 216 & 204 & 673 \\
\hline Wakayama & $\%$ & 30.9 & 100.0 & 29.2 & 100.0 & 30.3 & 100.0 \\
\hline 31 & $\mathrm{~N}$ & 154 & 703 & 56 & 240 & 210 & 943 \\
\hline Tottori & $\%$ & 21.9 & 100.0 & 23.3 & 100.0 & 22.3 & 100.0 \\
\hline 32 & $\mathrm{~N}$ & 77 & 387 & 27 & 189 & 104 & 576 \\
\hline Shimane & $\%$ & 19.9 & 100.0 & 14.3 & 100.0 & 18.1 & 100.0 \\
\hline 33 & $\mathrm{~N}$ & 171 & 1,501 & 83 & 577 & 254 & 2,078 \\
\hline Okayama & $\%$ & 11.4 & 100.0 & 14.4 & 100.0 & 12.2 & 100.0 \\
\hline 34 & $\mathrm{~N}$ & 419 & 1,392 & 185 & 678 & 604 & 2,070 \\
\hline Hiroshima & $\%$ & 30.1 & 100.0 & 27.3 & 100.0 & 29.2 & 100.0 \\
\hline 35 & $\mathrm{~N}$ & 212 & 894 & 108 & 427 & 320 & 1,321 \\
\hline Yamaguchi & $\%$ & 23.7 & 100.0 & 25.3 & 100.0 & 24.2 & 100.0 \\
\hline 36 & $\mathrm{~N}$ & 543 & 1,257 & 102 & 487 & 645 & 1,744 \\
\hline Tokushima & $\%$ & 43.2 & 100.0 & 20.9 & 100.0 & 37.0 & 100.0 \\
\hline 37 & $\mathrm{~N}$ & 130 & 536 & 52 & 208 & 182 & 744 \\
\hline Kagawa & $\%$ & 24.3 & 100.0 & 25.0 & 100.0 & 24.5 & 100.0 \\
\hline 38 & $\mathrm{~N}$ & 44 & 225 & 9 & 103 & 53 & 328 \\
\hline Ehime & $\%$ & 19.6 & 100.0 & 8.7 & 100.0 & 16.2 & 100.0 \\
\hline 39 & $\mathrm{~N}$ & 45 & 185 & 18 & 82 & 63 & 267 \\
\hline Kochi & $\%$ & 24.3 & 100.0 & 22.0 & 100.0 & 23.6 & 100.0 \\
\hline 40 & $\mathrm{~N}$ & 1,430 & 5,857 & 624 & 2,755 & 2,054 & 8,612 \\
\hline Fukuoka & $\%$ & 24.4 & 100.0 & 22.6 & 100.0 & 23.9 & 100.0 \\
\hline 41 & $\mathrm{~N}$ & 94 & 313 & 40 & 158 & 134 & 471 \\
\hline Saga & $\%$ & 30.0 & 100.0 & 25.3 & 100.0 & 28.5 & 100.0 \\
\hline 42 & $\mathrm{~N}$ & 333 & 1,415 & 153 & 678 & 486 & 2,093 \\
\hline Nagasaki & $\%$ & 23.5 & 100.0 & 22.6 & 100.0 & 23.2 & 100.0 \\
\hline 43 & $\mathrm{~N}$ & 576 & 1,594 & 262 & 731 & 838 & 2,325 \\
\hline Kumamoto & $\%$ & 36.1 & 100.0 & 35.8 & 100.0 & 36.0 & 100.0 \\
\hline 44 & $\mathrm{~N}$ & 238 & 819 & 105 & 425 & 343 & 1,244 \\
\hline Oita & $\%$ & 29.1 & 100.0 & 24.7 & 100.0 & 27.6 & 100.0 \\
\hline 45 & $\mathrm{~N}$ & 247 & 964 & 76 & 371 & 323 & 1,335 \\
\hline Miyazaki & $\%$ & 25.6 & 100.0 & 20.5 & 100.0 & 24.2 & 100.0 \\
\hline 46 & $\mathrm{~N}$ & 370 & 1,150 & 143 & 515 & 513 & 1,665 \\
\hline Kagoshima & $\%$ & 32.2 & 100.0 & 27.8 & 100.0 & 30.8 & 100.0 \\
\hline 47 & $\mathrm{~N}$ & 346 & 1,266 & 185 & 647 & 531 & 1,913 \\
\hline Okinawa & $\%$ & 27.3 & 100.0 & 28.6 & 100.0 & 27.8 & 100.0 \\
\hline & $\mathrm{N}$ & 20,483 & 82,624 & 7,779 & 33,244 & 28,262 & 115,868 \\
\hline All Japan & $\%$ & 24.8 & 100.0 & 23.4 & 100.0 & 24.4 & 100.0 \\
\hline
\end{tabular}


Table 3 Outcome at discharge stratified by existence of diabetes

(DPC “050050xx03x0xx”)

\begin{tabular}{lrrr}
\hline Outocome & Non-diabetic & Diabetic & \multicolumn{1}{c}{ Total } \\
\hline Recovered & 22,520 & 8,473 & 30,993 \\
& $97.0 \%$ & $96.9 \%$ & $97.0 \%$ \\
\hline No change & 441 & 173 & 614 \\
& $1.9 \%$ & $2.0 \%$ & $1.9 \%$ \\
\hline Worsened & 2 & 2 & 4 \\
& $0.0 \%$ & $0.0 \%$ & $0.0 \%$ \\
\hline Death due to Angina & 18 & 1 & 19 \\
& $0.1 \%$ & $0.0 \%$ & $0.1 \%$ \\
\hline Death due to other cause & 15 & 2 & 17 \\
& $0.1 \%$ & $0.0 \%$ & $0.1 \%$ \\
\hline Others & 219 & 89 & 308 \\
& $0.9 \%$ & $1.0 \%$ & $1.0 \%$ \\
\hline Total & 23,215 & 8,740 & 31,955 \\
& $100.0 \%$ & $100.0 \%$ & $100.0 \%$ \\
\hline
\end{tabular}

$\chi^{2}$ test: $\mathrm{p}=0.259$.

younger age than non-diabetic cases and consume more resources $(50,000$ JPY more both for male and female), although there was no significant difference in outcome at discharge. The largest difference in resource consumptions was observed for surgical intervention. Yosefy reported that diabetic patients tended to experience the intervention related complications, such as bleeding, surgical site infection, resurgery, etc ${ }^{7)}$. Artheriosclerosis and immunological dysfunctions are considered as base factors.

It is very interesting that there is a wide geographical variation among the diabetes prevalence among the observed population. The National Diabetes Survey also indicated that a higher diabetes prevalence was observed for Tokushima prefecture ${ }^{3)}$. Considering the relatively homogenous genetic characteristics of the Japanese, the differences in life style, especially diet related life style would explain this difference. Further epidemiological studies must be organized in order to clarify the mechanism of regional differences in prevalence.

In order to control the diabetic problems, the Ministry of Health, Labour and Welfare (MHLW) has introduced a new health promotion program called "Health checkups and healthcare advice with a particular focus on the metabolic syndrome" for the insured over 40 yr old. There is no doubt that diabetes has become one of the most important health threats for the Japanese. As we have suggested, this program will be useful if the proper strategy is employed ${ }^{8,9)}$. According to the results of our field study in Fukuoka prefecture, more than $40 \%$ of male inhabitants were obese and the prevalence of diabetes mellitus started to increase from $40 \mathrm{yr} \mathrm{old}^{10}$ ). This finding would support the validity of MHLW's strategy to start the preventive activity from $40 \mathrm{yr}$ old. However, the same study has clarified that the middle aged men did not participate at the health check-up and following health education because of the inconvenient time and places setting. Considering the importance of new disease management program targeting the diabetes mellitus, both of MHLW and individual insurer must re-organize the content of program.

Several limitations should be acknowledged. The DPC survey is conducted only between 1 July and 31 December every year, and data between 1 January and 30 June were not available. Another limitation is that our study does not cover all DPC hospitals. Therefore the number of cases was heterogeneous among the regions, i.e., a relatively small rate for Ehime prefecture was observed. Furthermore, there is a possibility of low report rate for diabetes as co-morbidity, because the existence of diabetes is not used for further DPC classification of angina pectoris. However, 
Table 4 Comparison of resource consumption between diabetic cases and non-doiabetic ones (DPC "050050xx03x0xx"; cost is described by "point". 1 point = $10 \mathrm{JPY}$ )

\begin{tabular}{|c|c|c|c|c|c|}
\hline & & & Male & & \\
\hline & Diabetes & $\mathrm{N}$ & Mean & SD & $\mathrm{p}$ value \\
\hline Age & No & 17,674 & 68.1 & 10.0 & $<0.001$ \\
\hline & Yes & 6,608 & 67.2 & 9.5 & \\
\hline Charged cost_Total & No & 17,674 & $140,314.0$ & $64,390.0$ & $<0.001$ \\
\hline & Yes & 6,608 & $145,206.9$ & $67,672.9$ & \\
\hline Charged cost_Consultation & No & 17,674 & 561.8 & 610.4 & $<0.001$ \\
\hline & Yes & 6,608 & 675.6 & 781.9 & \\
\hline Charged cost_Prescription & No & 17,674 & $1,348.2$ & $1,437.2$ & $<0.001$ \\
\hline & Yes & 6,608 & $1,588.2$ & $1,615.7$ & \\
\hline Charged cost_Injection & No & 17,674 & 759.9 & $2,980.1$ & 0.025 \\
\hline & Yes & 6,608 & 855.7 & $2,948.4$ & \\
\hline Charged cost Intervention & No & 17,674 & 144.7 & $1,415.9$ & 0.522 \\
\hline & Yes & 6,608 & 132.6 & 994.2 & \\
\hline Charged cost_Surgical intevention & No & 17,674 & $118,770.3$ & $56,325.3$ & $<0.001$ \\
\hline & Yes & 6,608 & $121,949.9$ & $58,952.2$ & \\
\hline Charged cost_labo-test & No & 17,674 & $4,155.2$ & $5,334.1$ & 0.793 \\
\hline & Yes & 6,608 & $4,175.2$ & $5,150.1$ & \\
\hline Charged cost_Diagnostic imaging & No & 17,674 & 516.1 & $1,192.5$ & 0.265 \\
\hline & Yes & 6,608 & 535.3 & $1,198.8$ & \\
\hline Chalson's Comorbity Index & No & 5,683 & 1.3 & 0.6 & $<0.001$ \\
\hline & Yes & 6,179 & 1.6 & 0.8 & \\
\hline Length of stay & No & 17,674 & 6.4 & 6.1 & $<0.001$ \\
\hline & Yes & 6,608 & 6.9 & 6.4 & \\
\hline
\end{tabular}

\begin{tabular}{|c|c|c|c|c|c|}
\hline & \multicolumn{5}{|c|}{ Female } \\
\hline & Diabetes & $\mathrm{N}$ & Mean & SD & $\mathrm{p}$ value \\
\hline \multirow[t]{2}{*}{ Age } & No & 5,541 & 73.0 & 8.7 & \multirow[t]{2}{*}{$<0.001$} \\
\hline & Yes & 2,132 & 71.4 & 8.5 & \\
\hline \multirow[t]{2}{*}{ Charged cost_Total } & No & 5,541 & $143,305.1$ & $67,635.0$ & \multirow{2}{*}{$<0.001$} \\
\hline & Yes & 2,132 & $149,252.1$ & $69,694.4$ & \\
\hline \multirow[t]{2}{*}{ Charged cost_Consultation } & No & 5,541 & 627.1 & 553.9 & \multirow[t]{2}{*}{$<0.001$} \\
\hline & Yes & 2,132 & 789.4 & 858.8 & \\
\hline \multirow[t]{2}{*}{ Charged cost_Prescription } & No & 5,541 & $1,463.6$ & $1,581.1$ & \multirow[t]{2}{*}{$<0.001$} \\
\hline & Yes & 2,132 & $1,691.1$ & $1,715.3$ & \\
\hline \multirow[t]{2}{*}{ Charged cost_Injection } & No & 5,541 & 950.6 & $3,447.2$ & \multirow[t]{2}{*}{0.535} \\
\hline & Yes & 2,132 & $1,047.0$ & $3,136.3$ & \\
\hline \multirow[t]{2}{*}{ Charged cost_Intervention } & No & 5,541 & 217.3 & $1,429.5$ & \multirow[t]{2}{*}{0.797} \\
\hline & Yes & 2,132 & 227.1 & $1,639.2$ & \\
\hline \multirow[t]{2}{*}{ Charged cost_Surgical intevention } & No & 5,541 & $117,776.7$ & $56,326.7$ & \multirow[t]{2}{*}{0.004} \\
\hline & Yes & 2,132 & $121,965.9$ & $57,557.0$ & \\
\hline \multirow[t]{2}{*}{ Charged cost_labo-test } & No & 5,541 & $4,734.8$ & $5,687.3$ & \multirow[t]{2}{*}{0.499} \\
\hline & Yes & 2,132 & $4,637.8$ & $5,460.2$ & \\
\hline \multirow[t]{2}{*}{ Charged cost_Diagnostic imaging } & No & 5,541 & 665.1 & $1,396.6$ & \multirow[t]{2}{*}{0.413} \\
\hline & Yes & 2,132 & 636.4 & $1,318.1$ & \\
\hline \multirow[t]{2}{*}{ Chalson's Comorbity Index } & No & 1,640 & 1.3 & 0.5 & \multirow[t]{2}{*}{$<0.001$} \\
\hline & Yes & 1,958 & 1.5 & 0.8 & \\
\hline \multirow[t]{2}{*}{ Length of stay } & No & 5,541 & 7.6 & 8.1 & \multirow[t]{2}{*}{0.002} \\
\hline & Yes & 2,132 & 8.3 & 7.8 & \\
\hline
\end{tabular}

$\mathrm{p}$ value: $t$-test, the normality of distributuion was checked by Levene's test. The modified $t$-test was employed for no-normal distribution cases. 
as the low report rate of diabetes would have the effect in direction to reduce the differences in resource consumptions, there would be more large differences in resource consumptions between the diabetic and nondiabetic cases. It is very important to recognize that the DPC database, one of the administrative data, is an important information sources for health policy making. It is expected that this precious data will be further used by various organizations in order to promote the nation's health.

\section{Acknowledgments}

This study was funded by Grants-in-Aid for Research on Policy Planning and Evaluation from the Ministry of Health, Labour and Welfare, Japan.

\section{References}

1) Ministry of Health, Labour and Welfare: Summary of vital statistics, http://www.mhlw.go.jp/english/ database/db-hw/populate/pop4.html (access 23 March 2010).

2) Ministry of Health, Labour and Welfare: National Medical Expenditures, http://www.mhlw.go.jp/ toukei/saikin/hw/k-iryohi/06/toukei1.html (access 23 March 2010).
3) Ministry of Health, Labour and Welfare: Patient Survey 2010, http://www.mhlw.go.jp/toukei/saikin/ hw/kanja/08/index.html (access 30 May 2010).

4) Ministry of Health, Labour and Welfare: National Health and Nutrition Survey 2008, http:// www.mhlw.go.jp/houdou/2008/04/h0430-2.html (access 30 May 2010).

5) Matsuda S: Introduction of DPC for clinicians, Tokyo: Jiho, 2009 (in Japanese).

6) Almdal T, Scharling H, Jensen JS, Vestergaard H: The independent effect of type 2 diabetes mellitus on ischemic heart disease, stroke, and death: a population-based study of 13,000 men and women with 20 years of follow-up. Archives of Internal Medicine 164, 1422-1426 (2004).

7) Yosefy C: Diabetic heart and the cardiovascular surgeon. Cardiovascular \& Hematological Disorders Drug Targets 8, 147-152 (2008).

8) Matsuda S, Fujino Y, Tanaka M: Development of IT based management system for health support program in Japan. APJDM 1, 83-89 (2007).

9) Matsuda S, Fujino Y, Tanaka Y, Haraguchi H: Development of Clinic based health promotion program in Kitakyushu, Japan. APJDM 2, 1-5 (2008).

10) National Health Insurance of Koge: Report on NHI Health up program. Fukuoka: Koge-machi, 2010 (in Japanese). 